\title{
Stability Analysis of Sampled-Data Teleoperation Systems
}

\author{
Ali Jazayeri and Mahdi Tavakoli \\ Department of Electrical and Computer Engineering, University of Alberta, Edmonton, AB T6G 2V4, Canada \\ Email: ali.jazayeri@ualberta.ca, tavakoli@ece.ualberta.ca
}

\begin{abstract}
In a haptic teleoperation system, closed-loop stability is influenced by the human operator and the environment dynamics, which are typically uncertain, time-varying or unknown. Therefore, in lieu of stability, the passivity of a teleoperation system as a sufficient condition for stability is investigated in the literature. The rationale for this is that, if the two-port network representing the teleoperator (comprising the master, the controller and communication channel, and the slave) is passive and is terminated to any passive but otherwise arbitrary operator and environment, the overall teleoperation system will also be passive. Instead of ensuring the passivity of the teleoperator in isolation, which is an overly-conservative requirement, in this paper we study the stability of the overall teleoperation system having assumed the passivity of the operator and the environment while permitting the teleoperator to be passive or nonpassive - such relaxation of the passivity condition on the teleoperator is expected to reduce design conservatism and allow for higher teleoperation performance. The broader aim of this study is to find the conditions for the stability of a teleoperation system when its controllers are implemented in discrete-time - it is known that discretization causes energy leaks and thus does not necessarily preserve passivity or stability. The absolute stability conditions for the sampled-data teleoperator are obtained using the Small Gain Theorem. The resulting condition for stability of the sampleddata teleoperation system imposes bounds on the controller parameters, the sampling period, and the master and slave robots damping terms.
\end{abstract}

\section{INTRODUCTION}

A teleoperation system consists of a user interface (master) and a remote robot (slave) interacting with a human operator and an environment, respectively. In bilateral teleoperation, while the slave robot mimics the master robot, the forces applied on the slave by the environment are displayed to the operator by the master. Teleoperation has applications ranging from telesurgery to material manipulation. Comprehensive studies on teleoperation can be found in [1], [2].

Teleoperation controllers are designed to meet two objectives: stability and transparency. Transparency can be defined as matching of positions and forces of the master and the slave, and ensures that the environment's impedance is transmitted to the operator with no distortion. There is a tradeoff between transparency and stability [3], [4].

To analyze the closed-loop stability of a teleoperation system, the operator and the environment models would have to be assumed known. While this assumption will

This research was supported by the Natural Sciences and Engineering Research Council (NSERC) of Canada under grants RGPIN-372042 and EQPEQ 375712 significantly simplify the stability analysis, it cannot be made in practice because the dynamic parameters of the human operator change in response to the specific requirements of the task at hand [5], and the dynamic parameters of the environment are also usually unknown, time-varying and/or nonlinear. On the other hand, after modeling the teleoperator as a two-port network [6], Llewellyn's theorem has been used for studying its absolute stability, which is equivalent to the overall teleoperation system stability for any passive but otherwise arbitrary operator and environment dynamics [7]. As a sufficient condition for stability, teleoperation system passivity has been studied using the scattering theory [8].

Another challenge in stability analysis of teleoperation systems is controller discretization. In teleoperation systems, the controllers are typically implemented in the discrete-time. The discretization of a stabilizing continuous-time controller, however, does not necessarily preserve stability of the closedloop system due to energy leaks in the zero-order hold [9], [10], [11]. A survey on sampled-data control for bilateral teleoperation can be found in [12].

Two-port network passivity of teleoperation systems has been extended to discrete-time using the Tustin method [13]. Also, [14] proposed a geometric approach that models all components of a sampled-data teleoperation system including both continuous-time and discrete-time parts in a unified Port-Hamiltonian form and adjusts the energy flow at the interconnection ports to preserve passivity of the system. A sampled-data teleoperation system preserves passivity after using six stable filters on the signals [11]. In addition, for discrete-time teleoperation stability, [15] has proposed boundaries on the controller gains and the sampling time.

The bilateral teleoperation problem has similarities with the virtual wall haptic rendering problem. In both problems, the user interacts with a master arm and feels forces due to either a virtual or physical environment. For a virtual wall, passivity approach has widely been applied for haptic interaction stability analysis. For instance, Colgate found a necessary and sufficient condition for passivity of a virtual wall under the assumption that the wall is implemented in the discrete-time domain while the operator is in the continuous-time domain [16]. Furthermore, for the discretetime haptic rendering problem, [17] derived boundaries on the stiffness of the virtual environment that are necessary and sufficient for the passivity of the virtual wall considering that the operator is acting as a mass-spring-damper model. Due to the conservativeness of the passivity, it has been 
tried to design non-passive but stable controllers. In [18], the energy of the system has been monitored by a passivity observer and a passivity controller makes the system nonpassive in some time intervals to reduce conservativeness and eventually to improve the performance of the discretetime haptic rendering system. No work to date has been reported concerning the requirements for absolute stability of a bilateral teleoperator considering the discretization effects.

In this paper, an absolute stability condition is developed for a discrete-time controlled teleoperator without assuming any model for the operator or the environment. Instead of ensuring the passivity of the teleoperator in isolation, which is an overly-conservative requirement, in this paper we study the stability of the overall teleoperation system having assumed the passivity of the operator and the environment while permitting the teleoperator to be passive or nonpassive. For this reason, the proposed absolute stability condition can be less conservative than ensuring the passivity of individual two-port networks that constitute the teleoperation system, thus allowing for higher transparency. We will find a general condition for stability of the overall teleoperation system. The condition can be used to verify the suitability of a discrete-time controller for a given teleoperation system.

This paper is organized as follows. A comparison between absolute stability and passivity of a continuous-time bilateral teleoperator is made in Section II. A sampled-data bilateral teleoperation system is modeled in Section III. This model is later used in Section IV to reach a condition for absolute stability of the sampled-data teleoperator. A few special cases that result in simpler conditions are considered in Section V, followed by concluding remarks in Section VI.

\section{Bilateral Teleoperation Stability - Passivity}

Passivity is defined based on energy dissipation at the port(s) of a network.

If the passivity condition is satisfied for each one-port network (the operator and the environment) and the twoport network representing the teleoperator (comprising the master, the controller, the communication channel and the slave), then their cascade interconnection will also be passive (Fig. 1). While this approach makes sure that each individual block is passive, in reality the overall teleoperation system passivity is possible even with a non-passive teleoperator. In fact, the best transparency in teleoperation can be reached when the teleoperator is non-passive [19]. Thus, the passivity framework has the limitation that, while passivity is a sufficient condition for stability of a system, the conservatism is furthere as passivity is ensured for individual subsystems in a cascade interconnection rather than for the overall system.

On the other hand, absolute stability of a two-port network is defined as its stability when it is coupled to passive but otherwise arbitrary one-port terminations (Fig. 1). Alternatively, a two-port network is absolutely stable iff the one-port network shown in Fig. 2 resulting from coupling the two-port network to a passive one-port network is passive.

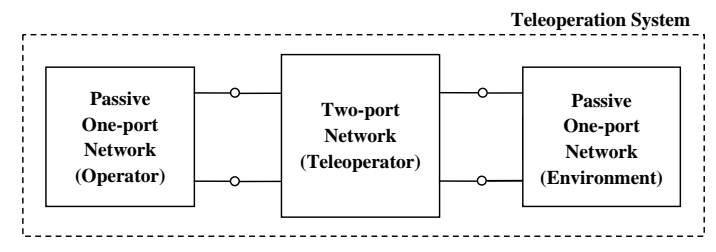

Fig. 1. Two-port network terminated to one-port networks. Passivity of each block will result in the passivity of the overall system while absolute stability will result in only stability of the overall system.

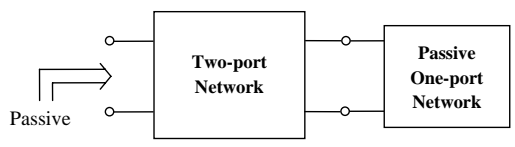

Fig. 2. Connecting a passive one-port network to any of the ports of a passive two-port network results in passivity of the remaining one-port networks

\section{SAMPled-Data TeleoperationModeling}

The dynamics of the master and slave robots are considered to be

$$
\begin{aligned}
f_{m}+f_{h} & =m_{m} \ddot{x}_{m}+b_{m} \dot{x}_{m} \\
f_{s}-f_{e} & =m_{s} \ddot{x}_{s}+b_{s} \dot{x}_{s}
\end{aligned}
$$

where $f_{h}$ is the interaction force between the master and the operator's hand, $m_{m}$ and $b_{m}$ are the master robot inertia and damping terms, respectively, and $x_{m}$ is the position of the master robot. Also, $f_{e}, m_{s}, b_{s}$ and $x_{s}$ are the slave and the environment counterparts. In the $s$-domain, (1) can be rewritten as

$$
\begin{aligned}
s X_{m} & =\frac{1}{m_{m} s+b_{m}}\left(F_{m}+F_{h}\right) \\
s X_{s} & =\frac{1}{m_{s} s+b_{s}}\left(F_{s}-F_{e}\right)
\end{aligned}
$$

The operator and the environment can be modeled as

$$
\begin{aligned}
\tilde{F}_{h}+F_{h} & =Z_{h} s X_{m} \\
\tilde{F}_{e}-F_{e} & =Z_{e} s X_{s}
\end{aligned}
$$

where $\tilde{F}_{h}$ and $\tilde{F}_{e}$ are the exogenous hand and environment forces, respectively. Also, $Z_{h}$ and $Z_{e}$ are the operator's hand and environment impedances, respectively.

Fig. 3 shows the sampled-data bilateral teleoperation system block diagram. The digital controller outputs $\left(f_{m}^{*}\right.$ and $\left.f_{s}^{*}\right)$ are converted to continuous-time $\left(f_{m}\right.$ and $\left.f_{s}\right)$ using Zero-Order-Hold ( $\mathrm{ZOH})$ with the transfer function $G_{h}(s)=$ $\left(1-e^{-s T}\right) / s$. To discretize the continuous-time signals, they are sampled at time instants separated by $T$. The ideal sampler unit generates the output $x^{*}(t)$ from the input $x(t)$ where

$$
x^{*}(t)=\sum_{k=0}^{\infty} x(k T) \delta(t-k T)
$$


The Laplace transform of the sampled signal is given by

$$
X^{*}(s)=\mathcal{L}\left\{x^{*}(t)\right\}=\sum_{k=0}^{\infty} x(k T) e^{-k T}
$$

The $z$-transform of the sampled signal is given by

$$
X(z)=\mathcal{Z}\left\{x^{*}(t)\right\}=\left.X^{*}(s)\right|_{s=1 / T \ln z}
$$

The master and the slave position output signals $\left(x_{m}\right.$ and $\left.x_{s}\right)$ are converted to discrete-time $\left(x_{m}^{*}\right.$ and $\left.x_{s}^{*}\right)$ using sampler blocks and fed to the controllers. The controllers deal only with discrete-time signals at their input and output. The controller output signals are converted to the continuous-time domain by the zero-order-holds:

$$
\begin{aligned}
F_{m}(s) & =\frac{1-e^{-s T}}{s} F_{m}^{*}(s) \\
F_{s}(s) & =\frac{1-e^{-s T}}{s} F_{s}^{*}(s)
\end{aligned}
$$

Assume that the teleoperation system uses a position error based (PEB) controller. As depicted in Fig. 3, the master and the slave position signals are transmitted to the other end through the communication channel. The PEB controllers work based on the master-slave position error in the discretetime domain and generate the following control signals $f_{m}^{*}$ and $f_{s}^{*}$ for the master and the slave:

$$
\begin{aligned}
F_{m}^{*}(s) & =C_{m}(z)\left[X_{s}^{*}(s)-X_{m}^{*}(s)\right] \\
F_{s}^{*}(s) & =C_{s}(z)\left[X_{m}^{*}(s)-X_{s}^{*}(s)\right]
\end{aligned}
$$

Now, (8) gives

$$
\begin{aligned}
F_{m}^{*}(s) & =C_{m}\left(e^{s T}\right)\left[-G_{m}^{*}(s) F_{m}^{*}(s)+G_{s}^{*}(s) f_{s}^{*}(s)\right] \\
F_{s}^{*}(s) & =C_{s}\left(e^{s t}\right)\left[-G_{s}^{*}(s) F_{m}^{*}(s)+G_{m}^{*}(s) f_{s}^{*}(s)\right]
\end{aligned}
$$

where $G_{m}^{*}(s)$ and $G_{s}^{*}(s)$ are discrete-time transfer functions and include dynamics of the sampler and the $\mathrm{ZOH}$. Indeed,

$$
\begin{aligned}
G_{m}^{*}(s) & =\frac{1}{T} \sum_{k} G_{m}\left(s+j k \omega_{T}\right) \\
G_{s}^{*}(s) & =\frac{1}{T} \sum_{k} G_{s}\left(s+j k \omega_{T}\right)
\end{aligned}
$$

where $\omega_{T}$ is the sampling frequency and is defined as $\omega=2 \pi / T$ and the continuous-time transfer functions for the master and the slave subsystems are

$$
\begin{aligned}
G_{m}(s) & =\frac{1}{m_{m} s+b_{m}+Z_{h}(s)} \cdot \frac{1-e^{-T s}}{s} \cdot \frac{1}{s} \\
G_{s}(s) & =\frac{1}{m_{s} s+b_{s}+Z_{e}(s)} \cdot \frac{1-e^{-T s}}{s} \cdot \frac{1}{s}
\end{aligned}
$$

\section{MAin Result}

In section III, the discrete-time teleoperation system was modelled and resulted in closed-loop equations (9). The main theorem for testing absolute stability of the sampled-data teleoperation system is given after the following lemma.

Lemma 1: The closed-loop characteristic equation of the sampled data teleoperation system shown in Fig. 3 does not depend on the input and output signals in the system and is

$$
1+C_{m}\left(e^{s T}\right) G_{m}^{*}(s)+C_{s}\left(e^{s T}\right) G_{s}^{*}(s)=0
$$

Proof:

It is easy to see that the transfer function from $\tilde{F}_{h}$ to $X_{m}$ is

$$
\frac{X_{m}(s)}{\tilde{F}_{h}(s)}=\frac{\left(1+C_{s}\left(e^{s T}\right) G_{s}^{*}(s)\right) H_{m}(s)}{1+C_{m}\left(e^{s T}\right) G_{m}^{*}(s)+C_{s}\left(e^{s T}\right) G_{s}^{*}(s)}
$$

where

$$
H_{m}(s)=\frac{1}{Z_{m}(s)+Z_{h}(s)} \cdot \frac{1}{s}
$$

and the master robot impedance is defined by $Z_{m}=m_{s} s+$ $b_{m}$. It can be shown that (12) is the also denominator of all transfer functions from inputs $\tilde{F}_{h}$ or $\tilde{F}_{e}$ to any other output.

Theorem 1: The sampled-data teleoperator resulting from using the discrete-time controller (8) for the continuous-time system represented by (1) as in Fig. 3 will be absolutely stable if

$$
\left\|M_{m} N_{m}+M_{s} N_{m}\right\|_{\infty}<1
$$

where

$$
\begin{aligned}
N_{m} & =\frac{2 b_{s} C_{m}\left(e^{s T}\right) r(s)}{2 b_{m} b_{s}+C_{m}\left(e^{s T}\right) b_{s} r(s)+C_{s}\left(e^{s T}\right) b_{m} r(s)} \\
N_{s} & =\frac{2 b_{m} C_{s}\left(e^{s T}\right) r(s)}{2 b_{m} b_{s}+C_{m}\left(e^{s T}\right) b_{s} r(s)+C_{s}\left(e^{s T}\right) b_{m} r(s)}
\end{aligned}
$$

$$
\begin{aligned}
M_{m}\left\{s, G_{m}^{*}\right\} & =-1+\frac{2 b_{m}}{r(s)} G_{m}^{*}(s) \\
M_{s}\left\{s, G_{s}^{*}\right\} & =-1+\frac{2 b_{s}}{r(s)} G_{s}^{*}(s)
\end{aligned}
$$

and

$$
r(j \omega)=\frac{T}{2} \frac{e^{-j \omega T}-1}{1-\cos \omega T}
$$

Proof:

For absolute stability, it is necessary and sufficient that (12) has all of its roots in the left half of the complex plane. The only assumption on the environment and the operator is that they are passive and as a result their impedances are 


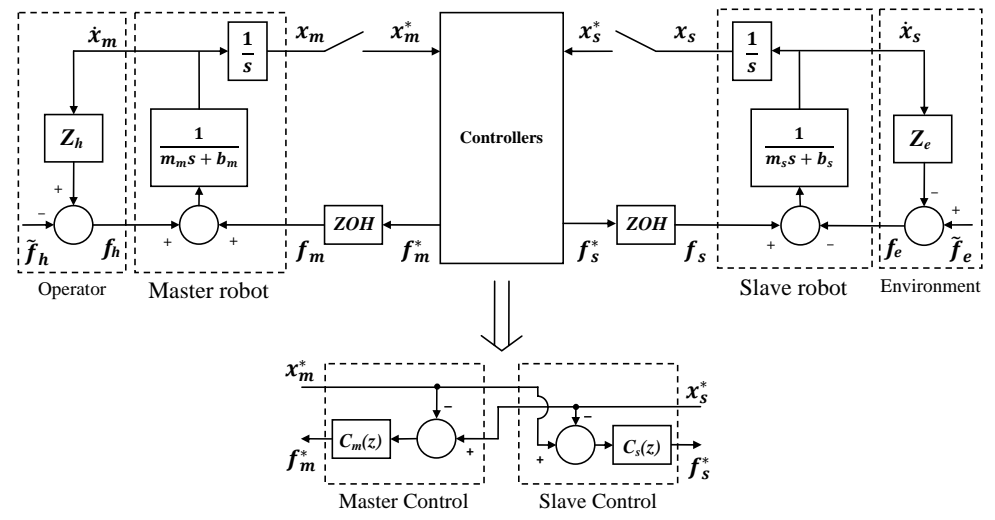

Fig. 3. Discrete-time controlled bilateral teleoperation system

positive real functions. The proof starts with the passivity assumption for the environment and the operator.

In the Nyquist plane, $Z_{h}$ and $Z_{e}$ cover the entire right half plane due to their positive-realness. Therefore, with positive $m_{i}$ amd $b_{i}$ we can say the following about the regions covered by each of the following functions:

$$
\begin{aligned}
1 /\left(m_{i} s+b_{i}+Z_{h}(s)\right) & \in \mathfrak{D}\left\{\frac{1}{2 b_{m}}, \frac{1}{b_{m}}\right\} \doteq \mathbb{D}_{m} \\
1 /\left(m_{i} s+b_{i}+Z_{e}(s)\right) & \in \mathfrak{D}\left\{\frac{1}{2 b_{s}}, \frac{1}{b_{s}}\right\} \doteq \mathbb{D}_{s}
\end{aligned}
$$

where $\mathfrak{D}\left\{x_{1}, x_{2}\right\}$ is a disk in the Nyquist plane with the center point of $x_{1}$ and the diameter of $x_{2}$. Such mappings of the environment and the operator in (19) are frequencyindependent. Now, (19) can be replaced in (11) to ultimately determine the regions of $G_{m}^{*}$ and $G_{s}^{*}$ in (10). Because $\mathbb{D}_{m}$ and $\mathbb{D}_{s}$ are frequency-independent, we can move them out of the summations in (10). The regions of $G_{m}^{*}$ and $G_{s}^{*}$ are, therefore,

$$
\begin{aligned}
G_{m}^{*} & \in r(j \omega) \mathbb{D}_{m} \\
G_{s}^{*} & \in r(j \omega) \mathbb{D}_{s}
\end{aligned}
$$

where $r(j \omega)$ is the frequency-dependent part in the summation (10) and can be calculated as

$$
r(j \omega)=\frac{1}{T} \sum_{k=-\infty}^{+\infty} \frac{1-e^{-\left(j \omega+j k \omega_{T}\right) T}}{\left(j \omega+j k \omega_{T}\right)^{2}}
$$

Simplification of (21) will result in (18). Consequently, the regions of $G_{m}^{*}$ and $G_{s}^{*}$ consist of a frequency-dependent part $r(j \omega)$ as in (18) and a frequency-independent part as in (19) that are shifted and scaled in the Nyquist plane. The areas in (20) can be mapped to the stable unit discs by the following transformations:

$$
\begin{aligned}
-1+\frac{2 b_{m}}{r(j \omega)} G_{m}^{*} & \in \mathfrak{D}\{0,2\} \\
-1+\frac{2 b_{s}}{r(j \omega)} G_{s}^{*} & \in \mathfrak{D}\{0,2\}
\end{aligned}
$$

Based on the transformations in (22), the linear fractional transformations (LFT) defined by $M_{m}$ and $M_{s}$ in (17) map the regions of $G_{m}^{*}$ and $G_{s}^{*}$ in (20) to two unit discs. Now, transformations $N_{m}$ and $N_{s}$ should be found such that the transformed system

$$
1+M_{m} N_{m}+M_{s} N_{s}=0
$$

has the same roots as the original characteristic equation (12). To do so, replacing $M_{m}$ and $M_{s}$ from (17) into (23) leads us to the condition

$$
\begin{array}{r}
1+\frac{-r(s)+2 b_{m} G_{m}^{*}}{r(s)} N_{m}\left\{s, G_{m}^{*}\right\} \\
+\frac{-r(s)+2 b_{s} G_{s}^{*}}{r(s)} N_{s}\left\{s, G_{s}^{*}\right\} \\
\quad=K\left(1+C_{m} G_{m}^{*}+C_{s} G_{s}^{*}\right)
\end{array}
$$

Note that (24) should be valid for any $G_{m}^{*}$ and $G_{s}^{*}$ and the coefficient $K$ should be independent of $G_{m}^{*}$ and $G_{s}^{*}$. By solving (24), the transformations $N_{m}$ and $N_{s}$ can be found as in (16). Finally, the Small Gain Theorem provides a sufficient condition for the stability of the characteristic equation (23) as given by (15).

In Theorem 1, transformations $M_{m}$ and $M_{s}$ are unit discs in the Nyquist plane. Condition (15) is the general condition that the controllers $C_{m}$ and $C_{s}$ should meet to ensure that the sampled-data controller teleoperation system is absolutely stable.

To achieve condition (15), it is sufficient to have $\left\|M_{m} N_{m}\right\|_{\infty}+\left\|M_{s} N_{s}\right\|_{\infty}<1$. Since $M_{m}$ and $M_{s}$ are unit discs in Nyquist plane, this sufficient condition for absolute stability reduces to

$$
\left\|N_{m}\right\|_{\infty}+\left\|N_{s}\right\|_{\infty}<1
$$

Equivalently, the sufficient condition for absolute stability can be expressed in terms of master and slave damping terms and controllers as 


$$
\frac{\left|2 b_{s} C_{m}\left(e^{j \omega T}\right) r(j \omega)\right|+\left|2 b_{m} C_{s}\left(e^{j \omega T}\right) r(j \omega)\right|}{\left|2 b_{m} b_{s}+C_{m}\left(e^{j \omega T}\right) b_{s} r(j \omega)+C_{s}\left(e^{j \omega T}\right) b_{m} r(j \omega)\right|}<1
$$

where $r(j \omega)$ is defined in (18). Note that (26) gives a general condition for absolute stability of sampled-data teleoperation systems, which is more conservative than (15). Also note that although absolute stability is less conservative than passivity, we have introduced some new conservativeness by addressing stability using the small-gain theorem.

\section{Special Cases}

Verifying (15) can be difficult in the general case for arbitrarily controllers. In this and the following subsections, condition (15) has been simplified for several special cases. In the following, assumptions regarding the control structure is made whereas (26) puts no assumption on it.

\section{A. Symmetric master and slave controllers}

A simplifying and realistic assumption is to design the controllers of the master and the slave to be proportional to the corresponding damping terms:

$$
\frac{C_{m}(z)}{C_{s}(z)}=\frac{b_{m}}{b_{s}}
$$

Replacing (27) in (16), $N_{m}$ and $N_{s}$ can be found as:

$$
N_{m}=N_{s}=\frac{C_{m}\left(e^{s T}\right) r(s)}{b_{m}+C_{m}\left(e^{s T}\right) r(s)}
$$

Thus, (28) and (25) can be combined to require the following for the absolute stability of the sampled-data teleoperator:

$$
\left\|N_{m}\right\|_{\infty}<\frac{1}{2}
$$

As a result, the absolute stability condition is

$$
\left|\frac{C_{m}\left(e^{j \omega T}\right) r(j \omega)}{b_{m}+C_{m}\left(e^{j \omega T}\right) r(j \omega)}\right|<\frac{1}{2}
$$

Let us define $\alpha$ and $\beta$ as

$$
\begin{aligned}
& \alpha=\Re\left\{C_{m}\left(e^{j \omega T}\right) r(j \omega)\right\} \\
& \beta=\Im\left\{C_{m}\left(e^{j \omega T}\right) r(j \omega)\right\}
\end{aligned}
$$

It is easy to show that

$$
\begin{aligned}
& \alpha=\frac{T}{2} \frac{1}{\cos \omega T-1} \Re\left\{\left(1-e^{-j \omega T}\right) C_{m}\left(e^{j \omega T}\right)\right\} \\
& \beta=\frac{T}{2} \frac{1}{\cos \omega T-1} \Im\left\{\left(1-e^{-j \omega T}\right) C_{m}\left(e^{j \omega T}\right)\right\}
\end{aligned}
$$

With these $\alpha$ and $\beta$, (30) can be simplified to

$$
b_{m}>-\frac{\alpha}{2}+\sqrt{\alpha^{2}+\frac{3}{4} \beta^{2}}
$$

which puts a lower bound on the master (and slave) damping. For a given controller $C_{m}\left(e^{j \omega T}\right)$, condition (35) can be checked to verify stability of the resulting sampled-data teleoperator system. Alternatively, condition (35) can be viewed as providing design guidelines in terms of selection of various control parameters and the sampling rate for stable teleoperation under discrete-time control.

\section{B. Known structure for controllers}

The stability condition (30) can be restated as

$$
\left|1+\frac{b_{m}}{r(j \omega) C_{m}\left(e^{j \omega}\right)}\right|>2
$$

In (36), the controller $C_{m}$ can be selected to have the following structure:

$$
C_{m}(z)=\frac{z^{-2}+2 z^{-1}-1}{2 z^{-1}-2} k_{1}
$$

where $k_{1}>0$ is a controller tuning parameter and we are interested in finding its admissible range for closed-loop stability. This controller in the $\omega$-domain is

$$
C_{m}\left(e^{j \omega T}\right)=\frac{e^{-2 j \omega T}+2 e^{-j \omega T}-1}{2 e^{-j \omega T}-2} k_{1}
$$

Now, replacing (38) into (36) yields

$$
\lambda^{2}+2 \lambda \cos \omega T+1>2 \lambda
$$

where $\lambda=T k_{1} /\left(2 b_{m}\right)$

The condition should be valid for all $\omega$ values. Thus for (39) to hold, it is sufficient that $\lambda^{2}-2 \lambda+1>2 \lambda$ or

$$
\frac{k_{1} T}{b_{m}}<2(2-\sqrt{3})
$$

which implies a lower bound on the master (and slave) damping for the stability of the sampled-data teleoperation system. Also, it places an upper bound on the controller gain and on the sampling period. This makes sense from a control perspective because generally as the controller gain and the sampling period increase, the stability margin of a sampleddata system shrinks.

In contrast to (27), another possibility for the controllers is

$$
\frac{C_{s}(z)}{k_{2}}=\frac{C_{m}(z)}{k_{1}}=\frac{z^{-2}+2 z^{-1}-1}{2 z^{-1}-2} ; k_{1}, k_{2}>0
$$

Replacing (41) into (26) reduces the absolute stability condition to

$$
b_{s} k_{1} T+b_{m} k_{2} T<\left|2 b_{m} b_{s} e^{j \omega T}+\frac{b_{s} k_{1} T}{2}+\frac{b_{m} k_{2} T}{2}\right|
$$

or

$$
\frac{k_{1} T}{2 b_{m}}+\frac{k_{2} T}{2 b_{s}}<\left|e^{j \omega T}+\frac{k_{1} T}{4 b_{m}}+\frac{k_{2} T}{4 b_{s}}\right|
$$

Thus, 


$$
2 \gamma<\left|e^{j \omega T}+\gamma\right|
$$

where

$$
\gamma=\frac{k_{1} T}{4 b_{m}}+\frac{k_{2} T}{4 b_{s}}
$$

It is easy to show that (44) can be simplified to

$$
2 \gamma<\gamma^{2}+2 \gamma \cos \omega T+1
$$

but (46) should be valid independent of frequency $\omega$. Therefore it is sufficient to have $2 \gamma<\gamma^{2}-2 \gamma+1$. As a result, the condition reduces to

$$
\frac{k_{1} T}{b_{m}}+\frac{k_{2} T}{b_{s}}<4(2-\sqrt{3})
$$

which again implies upper bounds on the sampling time and controller gains and lower gain on the master and slave damping terms.

The above were only two examples for selecting the controllers $C_{m}$ and $C_{s}$, and can be repeated for any given control structure to arrive at similar bounds in terms of the sampling period, robot damping and control gains such that the absolute stability of the resulting sampled-data teleoperator is ensured.

\section{Virtual wall}

The discrete-time controlled teleoperation system studied in this paper can be reduced to a discretely-simulated virtual wall that is haptically rendered for a user through a master user interface (no slave robot). It is easy to show that in this case the counterpart of the condition (35) on $b_{m}$ is

$$
b_{m}>-\alpha
$$

where $\alpha$ is given in (33). With a PD-type controller for the master, it can be shown that (48) is satisfied for all frequencies $\omega$ if the following condition is satisfied:

$$
b_{m}>\frac{K T}{2}+B
$$

where $K$ and $B$ are the proportional and derivative gains of the master's PD controller, which actually plays the role of the virtual coupling between the user and the infinitely-stiff virtual wall. This result is consistent with the condition given in [16] for the virtual wall haptic rendering problem.

\section{CONClusions AND Future Work}

In this paper, we found a condition for stability of a sampled-data teleoperation system using small gain theorem. Instead of requiring the passivity of the two-port network representing the teleoperator (i.e., the teleoperation system excluding the operator and the environment), we considered the closed-loop stability of the overall teleoperation system. Our contribution was to consider the impact of controller discretization on the stability of the teleoperation system.

The final condition for stability of the sampled-data system was simplified for certain controller structures, for which bounds were found on the controller parameters, the sampling time and the robot damping terms. Extending the proposed absolute stability criterion to the case of fourchannel teleoperation control (as opposed the position-error based control considered in this paper) remains as future work.

\section{REFERENCES}

[1] P. F. Hokayem and M. W. Spong, "Bilateral teleoperation: An historical survey," Automatica, vol. 42, no. 12, pp. 2035 - 2057, 2006.

[2] P. Arcara and C. Melchiorri, "Control schemes for teleoperation with time delay: A comparative study," Robotics and Autonomous Systems, no. 38, pp. 49-64, 2002.

[3] D. A. Lawrence, "Stability and transparency in bilateral teleoperation," IEEE Transactions on Robotics \& Automation, vol. 9, pp. 624-637, October 1993.

[4] A. Aziminejad, M. Tavakoli, R. Patel, and M. Moallem, "Transparent time-delayed bilateral teleoperation using wave variables," Control Systems Technology, IEEE Transactions on, vol. 16, pp. $548-555$, may 2008.

[5] Y. Matsuoka and R. Howe, "Hand impedance change during learning of a novel contact task," in World Congress on Medical Physics and Biomedical Engineering, 2000.

[6] B. Hannaford, "A design framework for teleoperators with kinesthetic feedback," IEEE Transactions on Robotics and Automation, vol. 5, pp. 426-434, 1989.

[7] S. Haykin, Active Network Theory. Reading, MA: Addison-Wesley, 1970.

[8] R. J. Anderson and M. W. Spong, "Asymptotic Stability for Force Reflecting Teleoperators with Time Delay," The International Journal of Robotics Research, vol. 11, no. 2, pp. 135-149, 1992.

[9] R. Gillespie and M. Cutkosky, "Stable user-specific rendering of the virtual wall," in Proceedings of the ASME International Mechanical Engineering Conference and Exposition, vol. 58, (Atlanta, GA), pp. 397-406, November 1996.

[10] J. Artigas, C. Preusche, G. Hirzinger, G. Borghesan, and C. Melchiorri, "Bilateral energy transfer in delayed teleoperation on the time domain,” pp. $671-676$, may 2008.

[11] G. Leung and B. Francis, "Bilateral control of teleoperators with time delay through a digital communication channel," in Proceedings of the Thirtieth Annual Allerton Conference on Communication, Control and Computing, pp. 692-701, 1992.

[12] J. Sheng and P. Liu, "A review of bilateral sampled-data control of teleoperators," pp. 385 -390, aug. 2004.

[13] R. Anderson, "Building a modular robot control system using passivity and scattering theory," in Proceedings of IEEE International Conference on Robotics and Automation, vol. 1, (Minneapolis, MN), pp. 698-705, April 1996.

[14] S. Stramigioli, C. Secchi, A. van der Schaft, and C. Fantuzzi., "A novel theory for sample data system passivity," in Proceedings of the IEEE/RSJ International Conference on Intelligent Robots and Systems, (Lausanne, Switzerland), pp. 1936-1941, 2002.

[15] M. Tavakoli, A. Aziminejad, R. Patel, and M. Moallem, "Discretetime bilateral teleoperation: modelling and stability analysis," Control Theory and Applications, IET, vol. 2, pp. 496-512, June 2008.

[16] J. Colgate and G. Schenkel, "Passivity of a class of sampled-data systems: Application to haptic interfaces," Journal of Robotic Systems, vol. 14 , no. 1 , pp. 37-47, 1997.

[17] J. Abbott and A. Okamura, "Effects of position quantization and sampling rate on virtual-wall passivity," Robotics, IEEE Transactions on, vol. 21, pp. 952 - 964, oct. 2005.

[18] J.-H. Ryu, B. Hannaford, C. Preusche, and G. Hirzinger, "Time domain passivity control with reference energy behavior," vol. 3, pp. 2932 2937 vol.3, oct. 2003.

[19] N. Diolaiti, G. Niemeyer, F. Barbagli, and J. Salisbury, "Stability of haptic rendering: Discretization, quantization, time delay, and coulomb effects," Robotics, IEEE Transactions on, vol. 22, pp. 256 -268, april 2006. 